\title{
Five Types of Ruled Helical Surfaces for Helical Conveyers, Support Anchors and Screws
}

\author{
S. N. Krivoshapko and Marina Rynkovskaya \\ RUDN University, Engineering Faculty, 117198, Miklukho-Maklaya Str., 6, Moscow, Russia
}

\begin{abstract}
The paper provides an overview of five types of ruled helical surfaces which can be used for helical conveyers, support anchors and screws. The geometry of helical surfaces has been well studied. Several available methods of strength analysis of helicoidal shells give one a choice in solving one-dimensional or two-dimensional problems. The basic problems considered in this paper include geometrical research and static analysis of right and developable helicoids. Several works dealing with stress-strain state of oblique, pseudo-developable helicoids, and pseudo-developable helicoid of general type are given in References.
\end{abstract}

\section{Introduction}

Geometry of helical surfaces has started to be investigated in the 18th century. An important contribution to the theory of helical surfaces was made by L. Euler (Switzerland), G. Monge (France), J. Meusnier, E. Catalan (France), G. Darboux, D. Hilbert (Germany), and many others.

The peak of publications on analytical methods of linear analysis of helicoidal shells was in the 50s. Professor S.N. Krivoshapko [1] described some theoretical methods of static analysis of engineering structures in the form of right and oblique helicoids. Later, in a paper [2] with 181 references, the detailed analysis of the existing methods for calculating of the helicoidal thin shells was presented, as well as the issues of geometric design and profiling of the helical surfaces. The study of the existing scientific literature showed that currently the engineers know only right and oblique helicoids, although an encyclopedia [3] provides information about the five types of ruled helical surfaces (Fig. 1).

Mechanical engineering is the main sphere of application of helicoidal shells.

\section{Methods of geometrical research}

Right helicoid is a minimal surface and its Gaussian curvature is negative (Fig. 1, a).

Oblique helicoid is the second well-known helical surface having rectilinear generatrixes inclined with respect to the axis of the helicoid at an angle of $\alpha<\pi / 2$ and moves remaining parallel to the generatrixes of a director cone's surface (Fig 1,b):

$$
\begin{aligned}
& x(u, v)=u \sin \alpha \cos v, \\
& y(u, v)=u \sin \alpha \sin v, \\
& z(v)=b v+u \cos \alpha,
\end{aligned}
$$
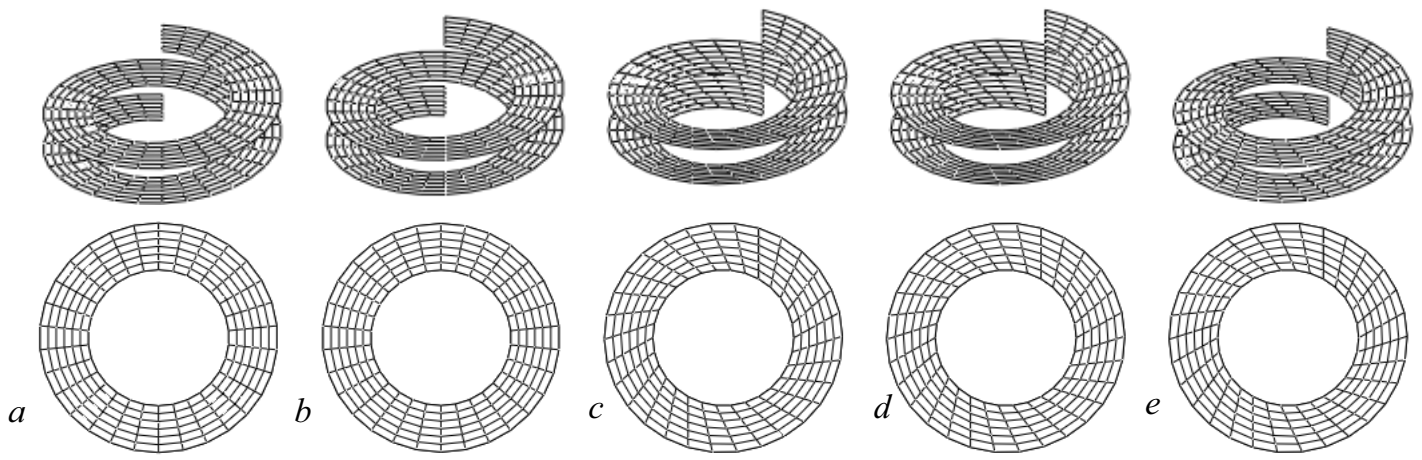

Figure 1. $(a$-right helicoid; $b$-oblique helicoid; $c$-developable helicoid; $d$-pseudo-developable helicoid of general type offered by S.F. Pilipaka; $e$ - pseudo-developable helicoid). 
where $|u|$ is the distance the axis from the selected point taken along the straight inclined generatrix.

The third ruled helical surface is called a developable helicoid (Fig. 1,c) and it has the parametric equations in the form:

$$
\begin{aligned}
& x=x(u, v)=a \cos v-\frac{a u \sin v}{m}, \\
& y=y(u, v)=a \sin v+\frac{a u \cos v}{m}, \\
& z=z(u, v)=b v+\frac{b u}{m}, \\
& m=\sqrt{a^{2}+b^{2}}
\end{aligned}
$$

So the curvilinear coordinate $u$ (or $v=$ const) is a rectilinear generatrix, tangent to the helical cuspidal edge, but lines $v(u=$ const $)$ are helixes. The curvilinear coordinates $u, v$ are parametric non-orthogonal conjugate curves. If we take a plane circular area with inside radius $a_{0}$ and cut it along a tangent to the inside contour then this circle area can be transformed into any open helicoid with a helical cuspidal edge lying on a cylinder with radius $a$, where $a=a_{0} \cos ^{2} \varphi$, here $\varphi$ is the helix angle.

S. N. Krivoshapko proposed a new form of presentation of the parametric equations of developable helicoid:

$$
\begin{aligned}
& x=x(u, s)=a_{0} \cos ^{2} \varphi\left(\cos \frac{s}{m}-\frac{u}{m} \sin \frac{s}{m}\right), \\
& y=y(u, s)=a_{0} \cos ^{2} \varphi\left(\sin \frac{s}{m}+\frac{u}{m} \cos \frac{s}{m}\right), \\
& z=z(u, s)=(s+u) \sin \varphi, m=a_{0} \cos \varphi,
\end{aligned}
$$

$u, s$ are curve non-orthogonal conjugate coordinates on the surface. The arc length $\mathrm{s}$ of a helical cuspidal edge can be determined by the formula $s=m v$.

For the fourth helical surface (pseudo - developable helicoid of general type), parametric equations were obtained by S.F. Pilipaka [4]. However, the authors believe that this surface is better to set in the following form (Fig. 1, d):

$$
\begin{aligned}
& x=a \cos v-u \cos \varepsilon \sin v, \\
& y=a \sin v+u \cos \varepsilon \cos v, \\
& z=b v+u \sin \varepsilon,
\end{aligned}
$$

where $\varepsilon$ is the angle of the the straight generatrix of the surface with the horizontal plane.

Fifth helical ruled surface which is defined by parametric equations (Fig. 1,e):

$$
\begin{aligned}
& x(u, v)=a \cos v-u \sin v, \\
& y(u, v)=a \sin v+u \cos v, \\
& z(v)=b v
\end{aligned}
$$

can be considered to be a special case of the surface (4) when $\varepsilon=0$. The surface (5) is a helical trace of uniform motion of horizontal rectilinear generatrix on the given helical guideline. Moreover, the straight generatrixes on the plane will coincide with the tangents to the helical guideline as well as in the case of the developable helicoid.
All these helical ruled surfaces are used in screw conveyors, the full length of which can reach $76 \mathrm{~m}$, screw support anchors, and in screws of different purposes.

\section{Methods of strength analysis}

It should be noted that geometry of these helical surfaces is studied quite well, but the analysis of the stress-strain state of thin shells with middle surfaces in the form of these ruled helicoids still cause some difficulties.

The first calculations of right helicoidal shells were presented by L.I. Solomon, V.G. Rekach and J.W. Cohen [5]. At present time, strength analysis is studied by M. Rynkovskaya [6], who found a number of inaccuracies in the method of V.G. Rekach and suggested the modified method for stress-strain analysis of long right helicoidal shells.

A. R. Yaroshenko [7] studied the axisymmetrical loading of an oblique helicoid given by formulas

$$
\begin{gathered}
x(r, \varphi)=r \cos \varphi ; y(r, \varphi)=r \sin \varphi ; \\
z=r \operatorname{ctg} \alpha+b \varphi,
\end{gathered}
$$

where $b=l /(2 \pi)$ and $l$ is the constant pitch of the helicoid. Substituting $\varphi=\psi-\operatorname{ctg} \alpha \cdot \operatorname{arctg}(r / b)$, he went on to an orthogonal non-conjugate system of curvilinear coordinates.

The coordinate lines in the accepted coordinate system are helixes and their orthogonal trajectories. A system of equilibrium equations was reduced to a system of ordinary differential equations. The sixth equation of equilibrium is satisfied identically.

At present, the calculations for determination of the stress-strain state of oblique helicoids are conducted by E Tupikova [8].

One-dimensional problem for shallow developable helicoid with two fixed helical sides was firstly solved by G.Ch. Bajoria [9], who took the shell material with a Poisson's ratio equal to zero $(v=0)$ in order to simplify the computation. His calculation is based on the system of three ordinary differential eighth-order equations with three unknown displacement parameters. The problem was solved numerically. Later, S.N. Krivoshapko and Kumudini Jayawardena [10] examined the onedimensional problem for a non-shallow thin shell in the form of long developable helicoid with several loops having arbitrary Poisson's ratio.

It was determined that the difference between the numerical results of calculations for $v=0.17$ and $v=0$ was, on average, about $10 \%$, but for the normal internal forces it was increased to $20 \%$.

Formulas (3) define a surface of the developable helicoid in non-orthogonal conjugate curvilinear coordinates. That is why, for the calculation of this type of helicoids it is necessary to use only the system of governing equations (six equilibrium equations, six geometrical and eight physical equations) of A.L. Gol'denveizer. But his equilibrium equations contain internal pseudo-forces and pseudo-moments, that is why, S.N. Krivoshapko suggested to work with the system of 
governing equations containing normal and shearing forces, bending and twisting moments reckoned per unit of curvilinear coordinates' length which are generally used in engineering practice:

Equilibrium equations:

$$
\begin{aligned}
& \frac{\partial}{\partial v}\left(A S_{v}\right)+\frac{N_{u}-N_{v}}{\sin \chi}\left(\frac{\partial B}{\partial u}-\frac{\partial A}{\partial v} \cos \chi\right)+\frac{\partial A}{\partial v} S_{u}+ \\
& +B \frac{\partial S_{u}}{\partial u} \cos \chi+B \frac{\partial N_{u}}{\partial u} \sin \chi-\frac{A B}{R_{u}} Q_{u}+A B X \sin \chi=0, \\
& \frac{\partial}{\partial v}\left(A N_{v}\right)+\frac{S_{u}+S_{v}}{\sin \chi}\left(\frac{\partial B}{\partial u}-\frac{\partial A}{\partial v} \cos \chi\right)-\frac{\partial A}{\partial v} N_{u}+ \\
& +B \frac{\partial S_{u}}{\partial u} \sin \chi-B \frac{\partial N_{u}}{\partial u} \cos \chi-\frac{A B}{\sin \chi}\left(\frac{Q_{v}}{R_{v}}-\frac{Q_{u}}{R_{u}} \cos \chi\right)+ \\
& +A B Y \sin \chi=0 \\
& \frac{N_{u}}{R_{u} \sin \chi}+\frac{N_{v}}{R_{v} \sin \chi}+\frac{1}{A B}\left[\frac{\partial}{\partial u}\left(B Q_{u}\right)+\frac{\partial}{\partial v}\left(A Q_{v}\right)\right]- \\
& -Z \sin \chi=0 \text {, } \\
& -\frac{\partial}{\partial v}\left(A M_{v}\right)+\frac{M_{u v}+M_{v u}}{\sin \chi}\left(\frac{\partial B}{\partial u}-\frac{\partial A}{\partial v} \cos \chi\right)+\frac{\partial A}{\partial v} M_{u}+ \\
& +B \frac{\partial M_{u}}{\partial v} \cos \chi+B \frac{\partial M_{u v}}{\partial u} \sin \chi+A B Q_{v} \sin \chi=0, \\
& \frac{\partial}{\partial v}\left(A M_{v u}\right)+\frac{M_{v}-M_{u}}{\sin \chi}\left(\frac{\partial B}{\partial u}-\frac{\partial A}{\partial v} \cos \chi\right)+\frac{\partial A}{\partial v} M_{u v} \\
& -B \frac{\partial M_{u}}{\partial u} \sin \chi+B \frac{\partial M_{u v}}{\partial u} \cos \chi+A B\left(Q_{u}+Q_{v} \cos \chi\right)=0, \\
& A B\left(Q_{u}+Q_{v} \cos \chi\right)=0, \\
& \left(S_{u}-S_{v}\right) \sin \chi+\left(N_{v}-N_{u}\right) \cos \chi+ \\
& +\frac{M_{u v}}{R_{u} \sin \chi}-\frac{M_{v u}}{R_{v} \sin \chi}=0 \text {, }
\end{aligned}
$$

geometrical equations derived by A.L. Gol'denveizer were taken by Krivoshapko without any modification; physical equations:

$$
\begin{gathered}
N_{v}=\frac{E h}{1-v^{2}}\left(\varepsilon_{v}-\varepsilon_{u v} \operatorname{ctg} \chi+v \varepsilon_{u}\right), \\
N_{u}=\frac{E h}{1-v^{2}}\left(\varepsilon_{u}-\varepsilon_{u v} \operatorname{ctg} \chi+v \varepsilon_{v}\right), \\
S_{v}=\frac{1-v}{2} C\left[\varepsilon_{u v}+\left(\varepsilon_{v}-\varepsilon_{u}\right) \operatorname{ctg} \chi\right], \\
S_{u}=\frac{1-v}{2} C\left[\varepsilon_{u v}+\left(\varepsilon_{u}-\varepsilon_{v}\right) \operatorname{ctg} \chi\right], \\
M_{v u}=\frac{E h^{3}}{12(1+v)}\left(\kappa_{v u}-\kappa_{u} \cos \chi\right), \\
M_{u v}=\frac{E h^{3}}{12(1+v)}\left(\kappa_{v u}-\kappa_{v} \cos \chi\right), \\
M_{v}=-\frac{E h^{3}}{12\left(1-v^{2}\right)}\left[\frac{\kappa_{u}+\kappa_{v}}{\sin \chi}-(1-v)\left(\kappa_{u} \sin \chi+\kappa_{u v} \operatorname{ctg} \chi\right)\right], \\
M_{u}=-\frac{E h^{3}}{12\left(1-v^{2}\right)}\left[\frac{\kappa_{u}+\kappa_{v}}{\sin \chi}-(1-v)\left(\kappa_{v} \sin \chi+\kappa_{u v} \operatorname{ctg} \chi\right)\right] .
\end{gathered}
$$

The positive direction of the forces and moments are shown in Fig. 2.

The hypothesis of non-interaction between layers of the shell which are "parallel" to the middle surface in the direction of its normal was used during the derivation of physical equations (7). The hypothesis show the fact that the normal component of the stress acting on the sections which are parallel to the middle surface is equal to zero.

Systems of equations (6), (7) in conjunction with geometrical equations of A.L. Gol'denveizer were used repeatedly by S.N. Krivoshapko and his disciples for the calculation of developable thin helicoidal shells loaded by axially symmetric load such as own weight [11].

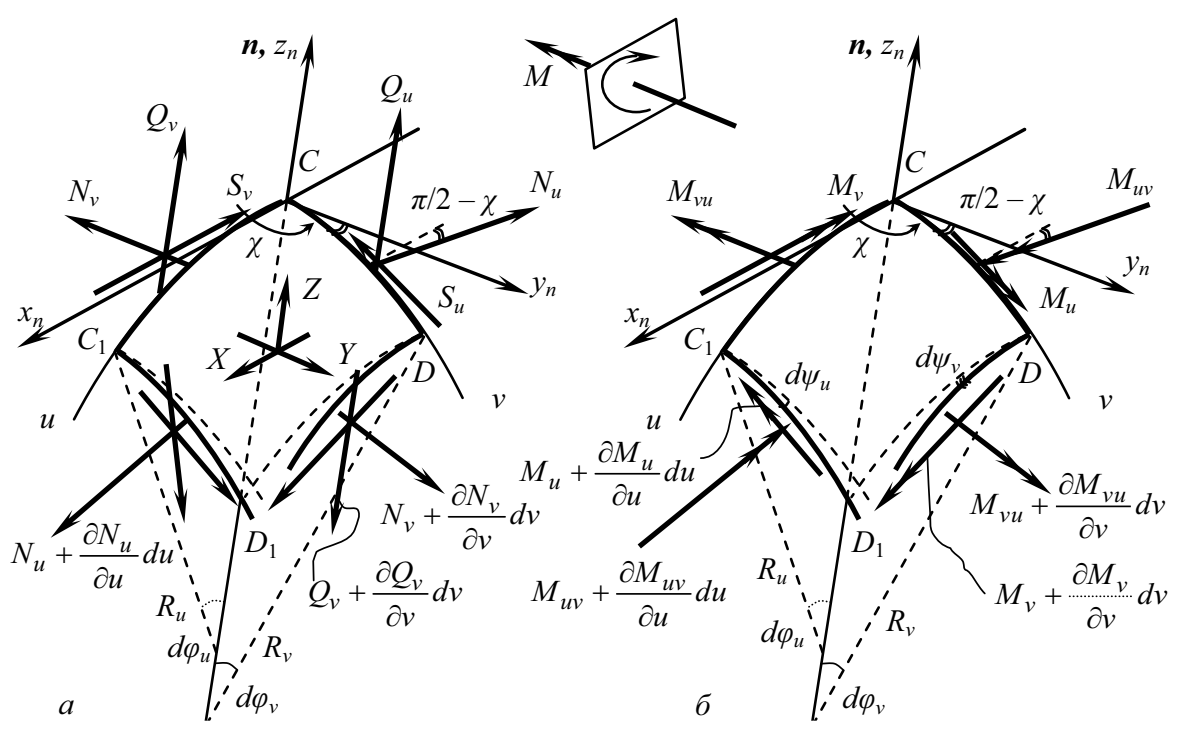

Figure 2. The positive direction of the inner forces and moments. 
For example, with the help of the asymptotic method of a small parameter, the proposed system of equations can be reduced to a system of three ordinary differential equations containing three unknown parameters of displacements that were presented in the series

$$
\begin{aligned}
& u_{1}=u_{1}(\alpha, \mu)=\sum_{i=0}^{\infty} H_{i}(\alpha) \mu^{i}, \\
& u_{2}=u_{2}(\alpha, \mu)=\sum_{i=0}^{\infty} V_{i}(\alpha) \mu^{i}, \\
& w=w(\alpha, \mu)=\sum_{i=0}^{\infty} W_{i}(\alpha) \mu^{i} .
\end{aligned}
$$

For the determination of the first members of the series (8), generating equations are used upon condition that the small parameter is equal to zero $(\mu=0)$. These generating equations are easily solved analytically. Solutions for the second and subsequent members of the series can be found similarly [11].

However, there were found some problems in analytical calculations of several integrals which could not be directly solved. The approximation of these integrals led to complicated calculations. M. Rynkovskaya's suggestion to use Bernoulli's numbers [12] for these integrals simplified the calculations and increased the practicability of the method.

The governing equations of Gol'denveizer will coincide with the equations $(6,7)$ of Krivoshapko if one uses the following relations:

$$
\begin{gathered}
N_{v}=N_{v}^{*} \sin \chi, \quad N_{u}=N_{u}^{*} \sin \chi, \\
S_{v}=-S_{v}^{*}+N_{v}^{*} \cos \chi, \quad S_{u}=S_{u}^{*}+N_{u}^{*} \cos \chi, \\
M_{v u}=M_{v u}^{*} \sin \chi, \quad M_{u v}=-M_{u v}^{*} \sin \chi, \\
M_{v}=-M_{v}^{*}+M_{v u}^{*} \cos \chi, \quad M_{u}=-M_{u}^{*}-M_{u v}^{*} \cos \chi .
\end{gathered}
$$

These relations connect internal forces and moments with pseudo-forces and pseudo moments denoted by the letters with stars, $\chi$ is the angle between the coordinate lines.

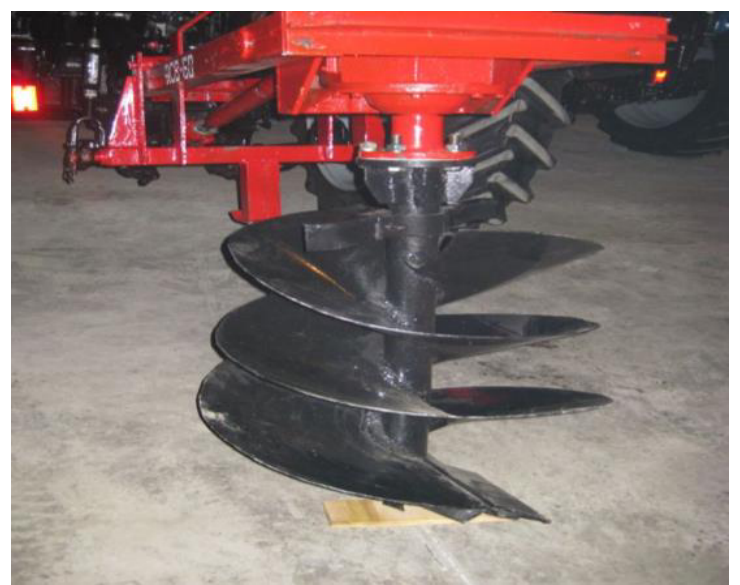

Figure 3. The hole-borer in the shape of developable helicoid.
Analytical solutions for the pseudo-developable helicoids of general form (Fig. 1, $d$ ) are not yet available, so in practice, it is necessary to carry out experimental research for each specific case.

A pseudo-developable helicoid was analyzed by S.M. Halabi [13], who solved some one-dimensional problems for this type of helicoid.

Various problems on the application of ruled helical surfaces, mostly right helicoids, are presented in [14-20]. The Fig. 3 shows the use of the developable helicoid as the hole-borer.

\section{Results and discussion}

The authors adduced some formulas for the examination of two types of ruled helicoidal thin shells that were used by them and by some other researchers for the determination of stress-strain state of long helicoids [21]. In engineering, right, oblique, developable and pseudodevelopable helicoids are widely used; however, their strength is determined in most cases on the basis of experiments. The monograph of D. Taylor [22] and article of D.Y. Panov [23] were the first two works devoted to the calculation of the strength of the right helicoidal shells in relation to parking structures. Right helicoids which are used in mechanical engineering are usually called twisted plate. This term describes the configuration better to most people, dealing with the calculation of compressor or turbine blades.

The example of using of the formulas presented in the paper can be demonstrated for the developable helicoids obtained by bending of the same annulus (Fig. 4).

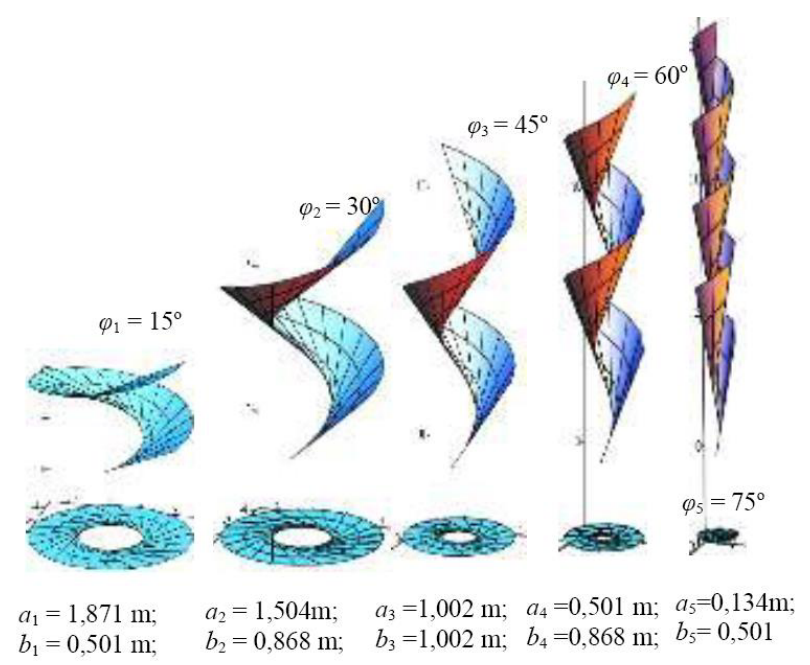

Figure 4. Developable helicoids obtained from the same annulus.

The mathematical apparatus of process of bending of a given annular plate into the helicoids with a given angle $\varphi$ of inclination of the rectilinear generatrixes was derived by S.N. Krivoshapko.

In the Fig. 5, the results of calculations of the reinforced concrete helicoidal shells with the middle surfaces presented in Fig. 4 are shown. The helicoids are rigidly fixed along the contour helical edges and are loaded by uniformly spread load: 


$$
X=-\frac{b g}{m}, Y=0, Z=\frac{a g}{m},
$$

where $g$ is a dead (own) weight of $1 \mathrm{~m}^{2}$ of the shell with the thickness of $10 \mathrm{~cm}$; Poison ratio is $0.17 ; a$ and $b$ are geometrical parameters of the middle surfaces shown in Fig. 4.

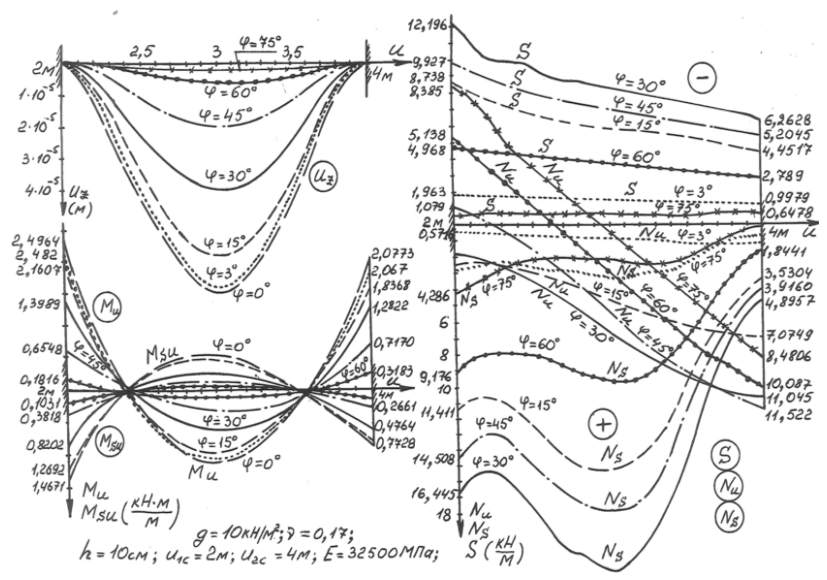

Figure 5. Results for the developed helicoids with various angles of generatrix inclination.

\section{Conclusion}

Although the subject of the geometry of ruled helical surfaces had its beginning in the eighteenth century, it still retains its attractiveness for an engineer.

Moreover, geometrical research on these surfaces is still in progress. In this paper, the authors described only analytical methods, numerical methods they used for comparison of results obtained by the both methods or for solution of the system of eight ordinary differential equations of the first order. It has been shown above that elastic behavior of right and developable helicoidal shells submitted to axisymmetric loading is well represented by various analytical methods.

\section{References}

1. Krivoshapko S.N. Helicoidal shells. Problems in theory and practice of engineering research, p.132136. (1998).

2. Krivoshapko S.N. Geometry and strength of general helicoidal shells, Appl. Mech. Rev., 52, No 5, p.161175 (1999).

3. Krivoshapko S.N., Ivanov V.N. Encyclopedia of Analytical Surfaces, Springer, 752 p. (2015).

4. Pylypaka S.F. Control of bending of ruled surfaces on an example of a screw conoid. Prikl. Geom. ta Inzh. Grafika, 70, p. 180-186 (2002).

5. Cohen J.W. On stress calculations in helicoidal shells and propeller blades. Holland, Walman, $100 \mathrm{p}$. (1955).

6. M.I. Rynkovskaya. Analytical Methods of Analysis of Right and Open Helicoids. Northeast Amer. Society of Eng. Educ. Conf., P.1-5 (2009).
7. Yaroshenko, A.R. Axe-symmetrical deformation of helicoidal shell with rectangular profile. Din. i Prochn. Mashin, 12, p. 3-9 (1971).

8. Tupikova E.M. Analysis of the thin elastic shells in the form of long oblique helicoid, Struct. Mech. of Eng. Constr. and Build., № 3, p. 23-27 (2015).

9. Badjoriya G.Ch. Analysis of long developable helicoid on moment theory in displacements. Struct. Mech. and Calcul. of Structures, 3, p.22-24 (1985).

10. Krivoshapko S.N., Kumudini D. Analysis of thin elastic shells in the form of Archimedes screw with the same angles of inclination of its generatrixes. Math. Probl. in Phys. and Techn., MFTI, p. 96-103 (1992).

11. Krivoshapko S.N. Geometry of linear surfaces with the return rib and linear theory for torso-helicoid calculation, RUDN, p.357 (2009).

12. M. Rynkovskaya, On application of Bernoulli's numbers for thin torso-helicoids calculated by asymptotic method of small parameter, MOO Spatial Structures, 12, p. 59-64 (2009).

13. Halabi, S.M. Moment theory of thin helicoidal pseudo-developable shells. Struct. Mech. of Eng. Constr. and Build., 10, p. 61-67 (2001).

14. Roberts A.W., Manjunath K.S., Mcbride W. The mechanics of screw feeder performance for bulk solids flow control. Nat. Conf. Publ., Inst. Eng., 92/7, P. 333-338 (1992).

15. Dabrowski Otton, Sapian Czeslaw. Loading of a central screw chute in a coal storage container. Pr. nauk. Inst. beed. pwrocl., 51, P. 125-130 (1987).

16. Teraoka Ats. Analisis del husillo de alta plastificacion para el moldeo por inyeccion. Rev. plast. mod., 46, № 463, P. 55-64 (1995).

17. Iura Masashi and Hirashima Masaharu. Fourier analysis of shallow right helicoidal shells. Trans. Jap. Soc. Civ. Eng., 14, P. 55-59 (1984).

18. Knabel J., Lewinski T. Selected equilibrium problem of thin elastic helicoidal shells. Arch. Civil Eng., 42(2), P. 245-257 (1999).

19. Kielb R.E., Leissa A.W., MacBain J.C. Vibrations of twisted cantilever plates - a comparison of theoretical results. Int. J. for NumericaL Meth. in Engineering, 21, P. 1365-1380 (1985).

20. Hoyashita Shigeru. Calculation method for profile of helical gear with tooth-trace modification finished by worm-shaped tool or form grinding wheel, Jap. Soc. Mech. Eng. C., 61, №583, P. 1149-1156 (1995).

21. Krivoshapko S.N., Gbaguidi A.G. Two methods of analysis of thin elastic open helicoidal shells.Int. Journ. of Res. and Rev.in Appl. Sc., 12, Iss.3, p.382390 (2012).

22. Taylor D.W. The Speed and Power of Ships. Ransdell Incorporated, Washington D.C., 130 p. (1933).

23. Panov D.Yu. Wind screw calculation [Raschet vozdushnogo vinta], Tr. TzAGI, 288 (1937). 